\title{
The inverted Muscle Skeleton approach: moving beyond rigid exoskeletons*
}

\author{
Martijn E. Grootens ${ }^{1 \star}$
}

\author{
Edsko E.G. Hekman ${ }^{1}$
}

\begin{abstract}
Exoskeleton technology has improved significantly over the past decades, yet devices are still not user-friendly. Recently, so-called exosuits-soft wearable suits that assist walking-have been proposed, but their torques are limited.

In this paper we propose the inverted Muscle Skeleton (iMS) approach; this method allows for the design of wearable robotics that are less rigid and more lightweight than exoskeletons, but more powerful than exosuits.

We have applied our approach to the design of a low-power knee orthosis that assists knee extension. Evaluation of the prototype during one-legged squats shows that the device can successfully generate knee extension torques that reduce muscle activity in the knee extensor muscles.

The approach is promising and we believe that higher joint torques can be generated with more powerful actuators. Future work includes testing the device during walking and applying the method to the design of orthoses for other joints.
\end{abstract}

\section{INTRODUCTION}

Exoskeleton technology has advanced considerably over the past decades. Robotic gait trainers, such as the Lokomat [1] and LOPES [2], are increasingly being used in rehabilitation clinics and a number of lower extremity exoskeletons, such as the ReWalk [3], Ekso GT [4], and Indego Personal [5], is becoming available for home use.

However, despite the improvements that have been made, exoskeletons remain bulky and heavy-although it is known that adding mass to the legs increases the metabolic cost of walking [6]. Exoskeletons require considerable donning times and the alignment of their joints with those of their wearer is critical, since misalignment may cause discomfort or injury [7]. In brief, exoskeletons are not yet user-friendly.

Some exoskeletons, such as the BLEEX [8], serve to relieve their wearer from loads. Most exoskeletons however, are used to apply joint torques. For this type, the provision of an external skeleton is not a goal per se. Considering the shortcomings of current exoskeletons and the fact that it is not required to use an exoskeleton to deliver joint torques, it is sensible to focus on more flexible solutions.

Over the past decade, various soft actuated orthoses have been developed for applications ranging from shoulder rehabilitation [9] to the treatment of gait pathologies [10], and more recently, so-called exosuits have been proposed: flexible robotic suits that are meant to assist human walking [11], [12]. These designs are created by following a biomimetic

\footnotetext{
*This work is supported by NWO, Domain Applied and Engineering Sciences (TTW), project no. 14429.

${ }^{1}$ Department of Biomechanical Engineering, University of Twente, 7522 NB Enschede, The Netherlands.

${ }^{\star}$ m.e.grootens@utwente.nl.
}

approach, in which artificial muscles act in parallel to the human muscles.

Current exosuits mainly focus on delivering assisting torques to the ankle and hip joints. Applications include assisting gait in healthy individuals [12], [13], improving gait in stroke survivors [14], and reducing the metabolic cost of walking [15]. However, exosuits cannot yet replace rigid exoskeletons, since the torques they generate are generally not larger than $40 \%$ of the torques required for human gait.

The joint torques delivered by exosuits are limited by their biomimetic design. Biological muscles exert large shear forces on the bones to which they are attached. Similar shear forces occur in exosuits, now due to the artificial muscles. These shear forces act directly on the skin and underlying tissue of the wearer of the suit, which means that the maximum joint torques are limited by the maximum shear force that the wearer of an exosuit can support [16].

In this paper, we investigate a new approach: we show that by not mimicking, but inverting biology, we can avoid loading the human body with excessive shear forces. Therefore, our inverted Muscle Skeleton (iMS) approach has the potential to deliver joint torques as high as those delivered by exoskeletons. We believe that our design approach can help to find an alternative to rigid exoskeletons.

\section{ThE INVERTED Muscle SKELETON APPROACH}

\section{A. The iMS approach}

In the human body, muscles generate tension forces between the bones of body segments in order to generate joint torques. Fig. 1(a) shows how the quadriceps muscles generate a knee extension torque and how the calf muscles generate an ankle plantar flexion torque. The muscle forces are almost collinear with the bones on which they pull and their moment arms are small. The required muscle forces are therefore very high and a large shear component (along the axes of the bones) loads the bones with high compression forces.

Exosuits based on a biomimetic design approach, such as those presented in [12] and [13], use muscle-like actuators that exert tension forces on the limbs of their wearer. Fig. 1(b) shows how a knee extension torque is generated by tensioning a cable between the proximal and distal leg segments and how an ankle plantar flexion torque is generated using a cable between the distal leg segment and the heel. Again, moment arms are small and the cables are almost collinear with the axes of the limbs. The cable forces need to be high in order to generate a significant joint torque, but this also means that the resulting shear forces will be high. Unfortunately, these high shear forces are acting on 
(a)

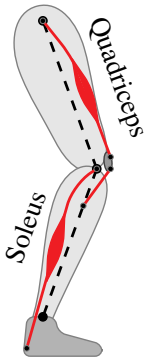

(b)

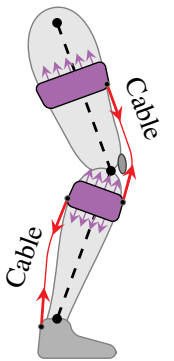

(c)

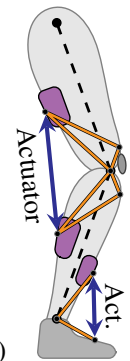

Fig. 1. Knee extension and ankle plantar flexion using (a) the human musculoskeletal system, (b) a biomimetic exosuit, and (c) the iMS approach.

the body of the wearer and, although they can be routed to locations such as the hips, they will cause discomfort. Furthermore, the high compliance of human tissue makes it difficult to keep the orthosis in place, which causes power losses and a reduction in control bandwidth [16].

We propose the iMS approach. By using actuators that provide compression-rather than tension-forces, the direction of the shear forces is inverted. Fig. 1(c) shows how linear actuators provide a knee extension and an ankle plantar flexion torque. Fundamental to the approach is the use of a network of cables to interconnect both actuator anchor points. These cables are loaded with the undesired shear forces and must cross through the joint axis when they go from one body segment to the other. The shear forces carried by the cables can either cancel each other, for example at the knee joint, or they can be transformed to compression forces on other body parts, such as the hips or sole of the foot.

Note that the key difference between the iMS approach and a 'pushing orthosis', such as the RoboKnee [17], is that the shear forces are carried by straps, rather than by a rigid frame. This allows for higher torques compared to exosuits, and a smaller size and mass compared to exoskeletons.

\section{B. Knee extension using the iMS approach}

We consider the knee orthosis that is shown in Fig. 1(c). The concept comprises a linear actuator, hinged to shells, interconnected by a network of straps. The network is symmetrical about the sagittal plane; it crosses over the leg, just above and below the patella. The actuator delivers a pushing force, so the orthosis generates an extension torque.

The parameters $h[\mathrm{~m}], d[\mathrm{~m}]$ and $h_{\mathrm{a}}[\mathrm{m}]$ that define the dimensions of the orthosis are shown in Fig. 2(a). We assume

(a)

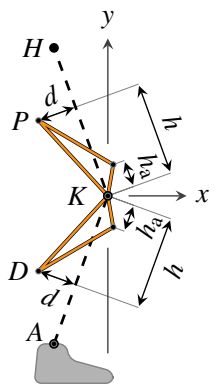

(b)

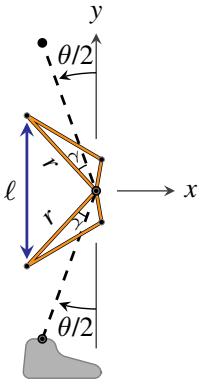

(c)

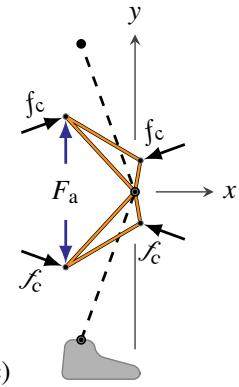

Fig. 2. Conceptual configuration for the knee orthosis: (a) anchor positions, (b) derived dimensions, (c) actuator and compression forces $F_{\mathrm{a}}$ and $f_{\mathrm{c}}$.

the configuration to be symmetrical about the bisector of $\angle H K A$. This means that the orthosis only exerts compression forces on the leg segments, since the shear components are carried by the cables and cancelled at the knee joint.

The actuator force $F_{\mathrm{a}}[\mathrm{N}]$, as shown in Fig. 2(c), needed for a knee torque $T \leq 0 \mathrm{Nm}$ at knee angle $\theta\left[{ }^{\circ}\right]$ (full knee extension corresponds with $\theta=0^{\circ}$ and the flexion direction is taken positive) is computed as

$$
F_{\mathrm{a}}(\theta, T)=-\frac{T}{d \cos (\theta / 2)+h \sin (\theta / 2)} .
$$

Fig. 2(c) shows the compression forces $f_{\mathrm{c}}[\mathrm{m}]$ that act on the leg both posteriorly (at the shells) and anteriorly (above and below the patella). They are equal and can be found as

$$
f_{\mathrm{c}}(T)=-\frac{T}{h-h_{\mathrm{a}}} \text {. }
$$

Pain Pressure Threshold (PPT) data (e.g. [18]) can be used to determine the surface area required to distribute the forces.

Note: Due to a difference in thickness of the proximal and distal leg segments and due to deformation of the soft tissue underlying the shells and anterior pads, it is not realistic to assume that all shear forces fully cancel. However, it is reasonable to assume that the major parts of the shear forces cancel out, so that only residual shear forces need to be transferred to the body. This can be done by connecting the proximal actuator hinge via a cable to a waist belt and the distal hinge via a cable to the shoe.

\section{PROTOTYPE DESIGN}

A low-power prototype, based on the concept from Section II-B, was constructed. It uses pneumatic cylinders, 3Dprinted shells, and a network of straps. This section describes the mechanical design, actuation, and control.

All stock components, such as cylinders, valves, and sensors, are listed in Table I.

\section{A. Mechanical design}

A detailed design is shown in Fig. 3. Two parallel pneumatic cylinders are used in order to increase the total actuator force and to avoid bending moments in the cylinder rods. Some components have been added, in order to keep the device in place and aid donning. Firstly, straps around the leg segments are added to keep the shells in contact with the

TABLE I

PNEUMATIC, SENSOR, AND CONTROL COMPONENTS

\begin{tabular}{ll}
\hline \hline Description & Type \\
\hline Festo & \\
\hline Cylinders 20/150 mm $(2 \times)$ & DSNU-20-150-P-A-S11 \\
Prop. dir. 5/3-way valve & MPYE-5-1/8-LF-010-B \\
Solenoid valve & VUVS-L20-M32C-MD-G18-F7-1C1 \\
Plastic tubing & PUN-8X1,25-SW \\
Push-in fittings & QS-G1/4-8, QSL-G1/8-8, and similar \\
Linear Hall sensor & SDAT-MHS-M160-1L-SA-E-0.3-M8 \\
Pressure sensors $(3 \times)$ & SPTE-P10R-S6-B-2.5K \\
\hline Other & \\
\hline Load cells $(2 \times)$ & Strain Measurement Devices S402 \\
DAQ card & National Instruments PCI-6229 \\
Real-time DAQ/control & MATLAB/Simulink Real-Time \\
\hline \hline
\end{tabular}




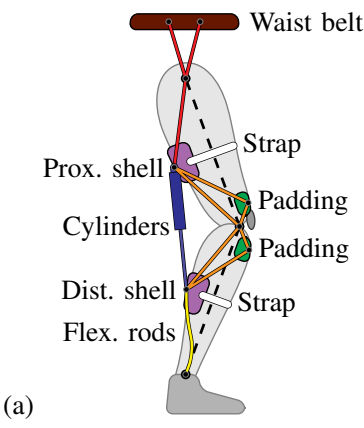

(b)

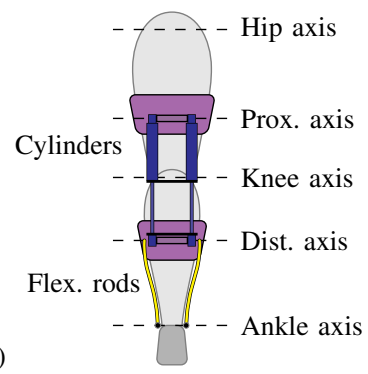

Fig. 3. Prototype schematic overview: (a) lateral view, (b) posterior view.

leg. Secondly, a waist belt is added, from which the mass of the upper part of the prototype is suspended. Finally, thin flexible rods with heim joints at their ends, are hinged to both sides of the shoe. The axes of the hinges are aligned with the ankle joint and the rods support the mass of the lower part of the prototype. The flexibility of the rods and the heim joints allow for unconstrained ankle movement.

Two cylinders with a $20 \mathrm{~mm}$ piston diameter, $8 \mathrm{~mm}$ rod diameter and $150 \mathrm{~mm}$ stroke are used. Each cylinder can generate a compression force of up to $235 \mathrm{~N}$ at the available absolute supply pressure of 8.5 bar. The choice for pneumatic cylinders was made based on their ease of use and off-theshelf availability. Although their need for a pressurized air supply makes them less suitable for untethered applications, they are suitable to demonstrate the principle.

The shells that are used to distribute the actuator force at the back of the leg are made from Polyamide using an SLS 3D-printing machine. Hardened steel axles pass through the shells, just behind the leg, and make up the rotation axes for the cylinders. The flexible part of the orthosis comprises $20 \mathrm{~mm}$ nylon straps, Velcro strips, elastic bands and padding. $\mathrm{Boa}^{\circledR}$ dials and laces are used to adjust and tighten the orthosis. All strap lengths can be adjusted using dials placed at the back of the shells and on the anterior padding.

Fig. 4 shows the final prototype, which has a mass of 1.5 $\mathrm{kg}$ (excluding shoes and external pneumatic components). The cylinders are spaced $120 \mathrm{~mm}$ apart and their hinges are placed at $h=0.215 \mathrm{~m}$ above and below the knee and at approximately $d=0.090 \mathrm{~m}$ behind the axes of the leg segments. The cylinders have a moment arm of $90 \mathrm{~mm}$ about the knee joint when the knee is stretched; an extension torque
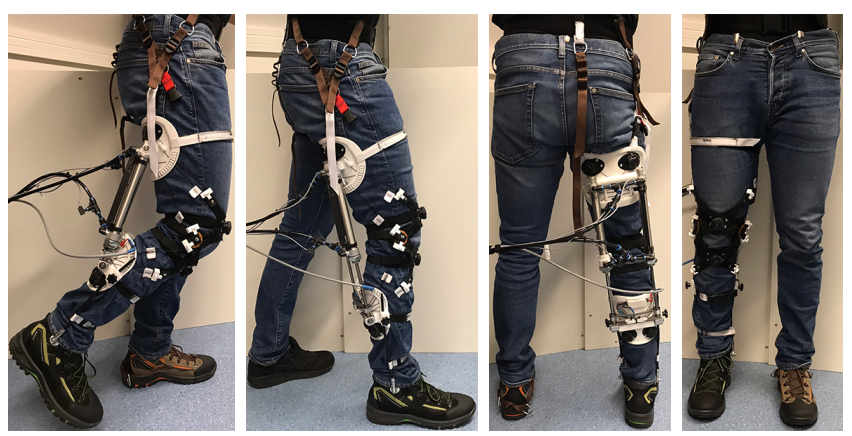

Fig. 4. Prototype realization of up to $-42 \mathrm{Nm}$ can then be delivered. The prototype allows for knee flexion up to $62^{\circ}$, which is sufficient for walking.

\section{B. Actuation}

Fig. 5 shows an overview of the pneumatic system.

A $50 \mathrm{~L}$ tank is used as pressure supply for the cylinders; a compressor maintains the pressure between 8.5 and 10 bar. A solenoid 3/2-way valve is used to connect and disconnect the prototype to and from the air supply; the prototype is disconnected from the pressure supply and exhausted to the atmosphere when any of the emergency buttons is pressed.

A proportional directional 5/3-way control valve is used to control the air flow to and from the cylinder. It controls both the flow rate and the direction: it either connects the extension chamber to the supply and the retraction chamber to the atmosphere, or the other way around.

All pneumatic tubing has an inner diameter of $5.7 \mathrm{~mm}$ and push-in fittings are used to interconnect all components.

\section{Sensing}

Two load cells, positioned inline with the pneumatic cylinders, are used to measure the total actuator force $F_{\mathrm{a}}=$ $f_{1}+f_{2}$ [N]. A linear Hall sensor is used to measure the piston position $\varepsilon[\mathrm{m}]$ at one of the cylinders. Two pressure sensors measure the pressure in the cylinder chambers and a third sensor measures the available supply pressure just before the control valve. All sensors are shown in Fig. 5.

The piston position $\varepsilon$ is taken as zero when the piston is fully retracted and extension is considered positive. The knee angle $\theta$ can now be estimated based on $\varepsilon$ and the dimensions of the prototype ( $\hat{\bullet}$ denotes the estimate of $\bullet$ ):

$$
\hat{\theta}(\varepsilon)=180^{\circ}-2 \gamma-2 \arcsin \left(\left(\ell_{0}+\varepsilon\right) /(2 r)\right),
$$

where $\gamma:=\arctan (d / h)\left[{ }^{\circ}\right]$ and $r:=\sqrt{d^{2}+h^{2}}[\mathrm{~m}]$, as shown in Fig. 2(b). Finally $\ell_{0}:=2 h-\varepsilon_{\max }[\mathrm{m}]$ is the minimum distance between the proximal and distal actuator hinges; $\varepsilon_{\max }=0.150 \mathrm{~m}$ is the cylinder stroke.

The actuator moment arm $R[\mathrm{~m}]$ about the knee joint can be estimated by

$$
\hat{R}(\varepsilon)=\sqrt{r^{2}-1 / 4\left(\ell_{0}+\varepsilon\right)^{2}}
$$

and the delivered joint torque $T[\mathrm{Nm}]$ can be estimated by

$$
\hat{T}\left(\varepsilon, F_{\mathrm{a}}\right)=-\hat{R}(\varepsilon) F_{\mathrm{a}} .
$$

Note that a positive actuator force results in a negative knee torque-an extension torque.

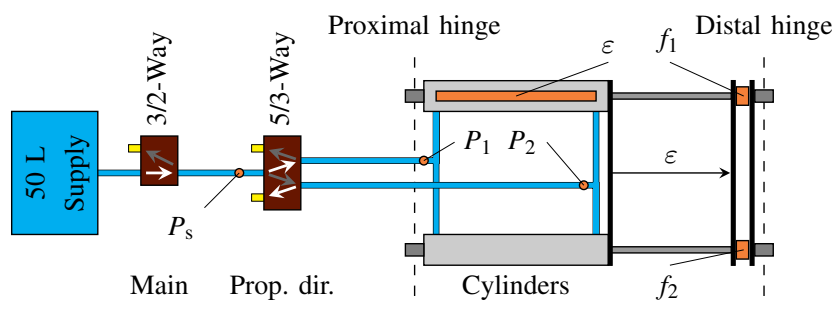

Fig. 5. Schematic overview of the pneumatic system with sensors (orange), valves (maroon), cylinders (grey), tubing (cyan), and exhausts (yellow). 


\section{Control}

The compressibility of air and the highly nonlinear behavior of air flow through pneumatic systems make position and force control of pneumatic cylinders difficult [19]. The nonlinear behavior of cylinders actuated by proportional control valves is modeled in [19] and a sliding mode force control strategy for such systems is presented in [20]. This strategy is adopted and uses all sensor data to control the cylinder force. The closed-loop system has both a reference force $F_{\mathrm{r}}[\mathrm{N}]$ and a reference force rate of change $\dot{F}_{\mathrm{r}}[\mathrm{N} / \mathrm{s}]$ as inputs. The friction of the cylinders is overcome by using the force measurements from the load cells instead of an estimate based on the chamber pressures.

Section III-C presented estimates of the knee angle and actuator moment arm based on the cylinder position. The desired actuator force can be computed based on a desired knee torque and these estimates.

Data acquisition and control are implemented using Matlab/Simulink R2016B. A target PC running Simulink RealTime is equipped with two National Instruments 6229 PCI data acquisition cards to interface with the hardware.

\section{PRototype EVAluation}

Testing the device during walking, for which it is intended, would require a gait assistance strategy, the effectiveness of which would greatly influence the results. We therefore decided to use one-legged squats as a device test, as we expected to see a reduction in extensor muscle activity if the device is effective in providing an assistive extension torque.

\section{A. Method}

Two healthy subjects performed multiple series of onelegged squats with the device worn on their right leg. A 0.5 $\mathrm{Hz}$ metronome was used to regulate the timing of the squats.

The subjects were instructed to keep their knee behind their toes; to move as smoothly as possible; to reverse the direction of motion at the beat of the metronome; and to use the hand rails only for balance. They were given a couple of minutes to practice and were asked to perform the squats over the full range of motion of the device.

A virtual prestressed compression spring was implemented on the prototype. The reference force and reference force rate of change that were given as inputs to the controlled orthosis are computed as follows:

$$
\begin{aligned}
& F_{\mathrm{r}}(\varepsilon)=f_{\text {pre }}+\varphi k_{\mathrm{s}}\left(\varepsilon_{\max }-\varepsilon\right), \\
& \dot{F}_{\mathrm{r}}(\dot{\varepsilon})=-\varphi k_{\mathrm{s}} \dot{\varepsilon},
\end{aligned}
$$

where $f_{\text {pre }}[\mathrm{N}]$ is the prestress of the virtual spring. (This prestress was set to $10 \mathrm{~N}$, so that the cylinders always provide a small force to compensate for the mass of the upper part of the prototype.) The virtual spring stiffness $k_{\mathrm{S}}[\mathrm{N} / \mathrm{m}]$ is subject specific and based on the body mass of the subject: $k_{\mathrm{s}}=\xi m_{\text {subject }}$. A value of $\xi=32 \mathrm{~N} / \mathrm{m} / \mathrm{kg}$ was chosen to ensure that we never require more force from the actuator than can be delivered (considering the minimum available supply pressure and maximum expected body mass). Finally, the gain $\varphi$ determines the amount of assistance being given.
The subjects performed squats at three levels of assistance - in randomized order and with a two-minute rest interval. The levels are: minimal impedance $(\varphi=0) ; 50 \%$ assistance $(\varphi=0.5)$; and $100 \%$ assistance $(\varphi=1)$. The subjects performed approximately 20 squats at each level.

A Delsys Trigno wireless EMG system was used to measure the muscle activity of the three knee extensor muscles of the right leg: the Rectus Femoris, Vastus Medialis, and Vastus Lateralis. All data was captured at $1000 \mathrm{~Hz}$.

Ethical approval was granted by the institutional review board and the participants gave written informed consent.

\section{B. Data processing}

The EMG data was processed using the following three steps: (1) 2nd order Butterworth high-pass filter with a cutoff frequency at $10 \mathrm{~Hz}$; (2) absolute value; (3) 2nd order Butterworth low-pass filter with a cut-off frequency at $1 \mathrm{~Hz}$.

Fig. 6 shows the cylinder position and both the reference force $F_{\mathrm{r}}$ and actual force $F_{\mathrm{a}}$ for subject 1 performing squats at assistance level $\varphi=1$. (Note that $\varepsilon=0 \mathrm{~m}$ corresponds to $\theta \approx 62^{\circ}$ and $\varepsilon=0.150 \mathrm{~m}$ to $\theta=0^{\circ}$. The scaling is almost linear.) We clearly see that the first couple of squats are not deep enough, because the subject is still getting used to the high level of assistance. There is also quite some variation in the motion profiles of the individual squats.

We can furthermore see that the actuator force $F_{\mathrm{a}}$ nicely tracked the reference force $F_{\mathrm{r}}$, but that the subject hit the cylinder end stop at $\varepsilon=0 \mathrm{~m}$ and 'sat' on it, thereby causing the peaks in $F_{\mathrm{a}}$. The subject never fully stretched his leg.

All the time series data was cut into separate blocks for each squat motion (identified by the tops in the time series of $\varepsilon$ and indicated in Fig. 6 by the shaded areas) and then resampled to a dimensionless time $t \in[0,1]$, to compensate for small differences in the timing of the squats.

For each assistance level, a combination of the 10 most similar (that is the combination with the lowest standard deviation) squat motions were selected from the squats that were recorded. In this way, the squats during which the

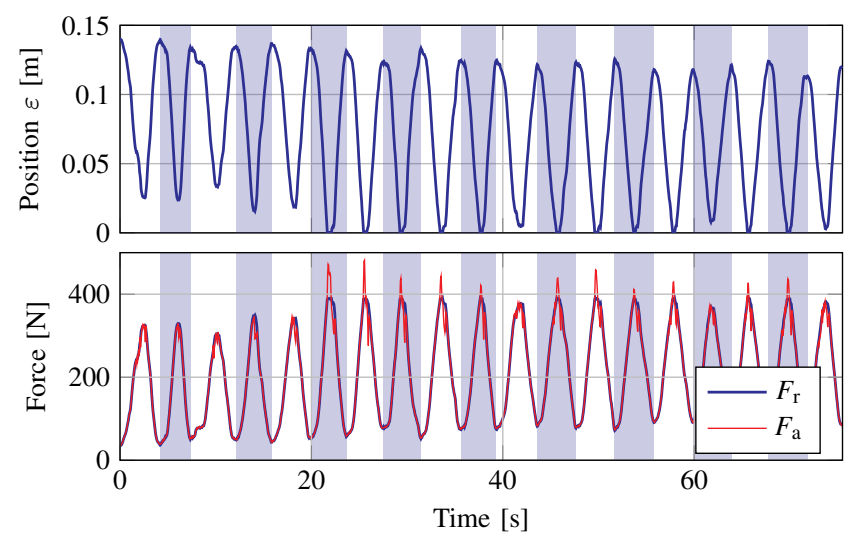

Fig. 6. Motion for subject 1 at the maximum assistance level of $\varphi=1$ the top graph shows the cylinder position $\varepsilon$; the bottom graph shows the reference force $F_{\mathrm{r}}$ and actuator force $F_{\mathrm{a}}$. The peaks in $F_{\mathrm{a}}$ show that the subject was 'sitting' on the end stop at $\varepsilon=0 \mathrm{~m}$. 

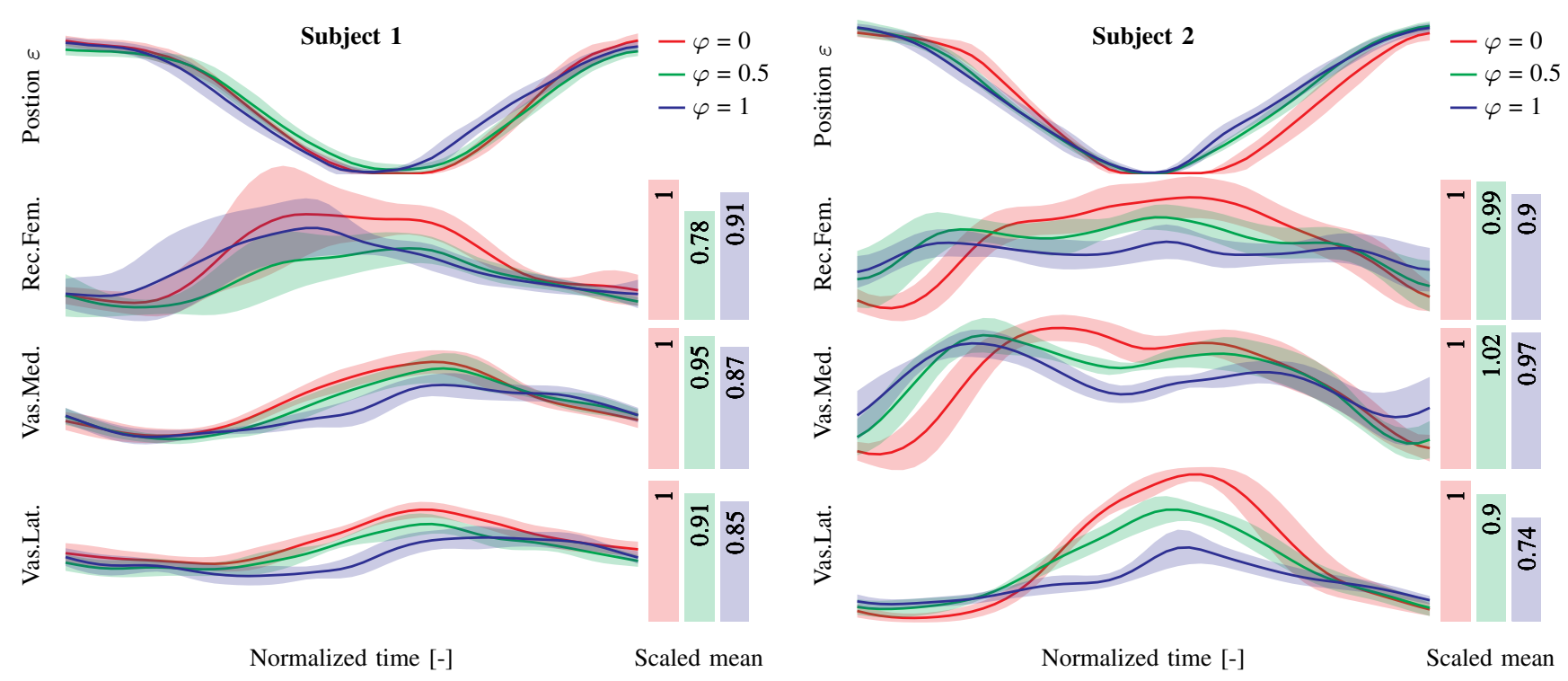

Fig. 7. Average data for 10 squats at $\varphi=0,0.5,1$ for both subjects. The top graph for each subject shows the cylinder motion and the following graphs show the extensor muscle activity. The thick lines denote the mean value and the shaded areas indicate the standard deviation. The bar graphs on the right show the mean value of the signals, scaled with the mean value of the $\varphi=0$ condition.

subject was still getting used to the level of assistance, or lost balance, were discarded.

\section{Results}

Fig. 7 shows the mean and standard deviation of the cylinder motion and EMG activity of both subjects, based on 10 squats at each assistance level.

We can clearly see, in particular for subject 2 , that the motion profile for $\varphi=0$ (no assistance) differs from the other two conditions. The subject is really 'sitting' on the end stop at $\varepsilon=0 \mathrm{~m}$ and the motion upwards is slightly delayed. This sitting on the end stop means that the subject is temporarily relieving his extensor muscles, so that the measured EMG activity is probably lower than it would have been without the device - this has no positive influence on our results.

The figures furthermore show that all three extensor muscles are relieved by the assistance given by the device. The EMG activity at half the normalized time, the moment at which the extensor muscles must deliver the highest force, is lower at $\varphi=0.5$ and even lower at $\varphi=1$.

The figure also shows bar graphs for the mean value of the muscle activity (that is the mean value of the mean muscle activity over 10 squats). The values are normalized with the mean value of the $\varphi=0$ (no assistance) condition, so that the relative muscle activity can be inspected.

We see that giving assistance induces a decrease in muscle activity. The exception being the Vastus Medialis of subject 2 , which shows $2 \%$ more activity at $\varphi=0.5$ than at $\varphi=0$. In general we also see that the muscle activity decreases with increasing assistive torque. The exception is the Rectus Femoris for subject 1 , which shows more activity at $\varphi=1$ than at $\varphi=0.5$. The exceptions may be due to the subject still getting used to the assistance or due to the one-legged squats being a rather difficult task (w.r.t. balance and effort).

\section{DISCUSSION}

We have used our iMS approach for the design of an actuated knee orthosis and have shown that the prototype can successfully apply assistive torques. We believe that it is possible to generate higher torques with stronger actuators.

\section{A. EMG reduction}

We implemented a virtual spring on the prototype to provide an assistive torque. The reduction in muscle activity due to the given assistance is evident and shows that the device can successfully deliver a knee extension torque. However, the assistance strategy is very simple and a more intelligent strategy, for instance based on inverse dynamics or EMG measurements, is likely to be more effective.

\section{B. Challenges of the iMS approach}

The prototype stays in place during squats, though keeping it in place during walking is not straightforward. Hip ab/adduction and endo-/exorotation vary the tension in the straps to the hip belt, so that the prototype slightly rotates about, and slides down along, the leg. It needs to be regularly adjusted and further design improvements are needed to keep the device in place.

\section{Application of the iMS approach to other joints}

Application of the iMS approach to the design of an orthosis for ankle plantar flexion, as is shown schematically in Fig. 1(c), is a logical next application of the approach. The design of an orthosis for ankle dorsiflexion also seems feasible, for example by using an actuator between a shell behind the lower leg and the shoe, just behind the heel.

The approach may also be applied to joints of the upper extremities; one can think of elbow extension, or finger extension in an orthosis for rehabilitation of patients with finger contractures. 


\section{Limitations of the iMS approach}

However, the iMS approach cannot be applied to all joints. For instance, it is not possible to generate knee flexion torques, as it is not feasible to place linear actuators anterior to the leg and keep them in place using only straps.

Furthermore, application to the hip joint seems unfeasible due to the large range of flexion/extension motion and the soft tissue around the stomach onto which compression forces cannot comfortably be exerted.

\section{E. Hybrid designs}

Combining designs based on the iMS principle and biomimetic designs may be an interesting solution to overcome some of the limitations of the iMS approach. Considering the knee for instance, the addition of a hip belt and connections to the ankle (as already present in the prototype) allows for the actuators to also deliver a tension force. This means that the same device can also generate a knee flexion torque, which could be used to provide assistance during the swing phase of gait.

\section{F. Comparison to exoskeletons and exosuits}

If we compare designs based on the iMS approach to those based on exoskeleton technology, then the former can become less bulky, more lightweight, less constraining and less conspicuous than the latter. However, exoskeletons still can be more powerful than designs based on the iMS approach, because their torques are not necessarily dependent on user comfort and because they can transfer loads directly to the ground.

Compared to exosuits, designs based on the iMS approach do contain some rigid components (actuators and shells), but in turn exert no shear forces on their wearer. The iMS approach thus allows for the design of actuated orthoses that can provide higher joint torques than exosuits, yet are less rigid and constraining than exoskeletons.

\section{CONCLUSIONS AND FUTURE WORK}

We have proposed the new iMS approach; it may be taken during the design of flexible wearable robotics that can deliver higher joint torques than biomimetic alternatives.

We have successfully applied the principle to the design of an actuated knee orthosis and evaluation during one-legged squats shows that the prototype can deliver assistive knee extension torques that induce a reduction of knee extensor muscle activity in the wearer of the device.

Future updates to the design include increasing the range of motion and joint torque that can be delivered. Furthermore, user comfort and ease-of-use can be improved and size and weight can be optimized. Future experiments include testing the device during walking and characterization of the forces that act on the wearer of the device.

\section{ACKNOWLEDGEMENTS}

We thank Q. Meinders for manufacturing the prototype and G.V. Durandau for helping with the experiments.

\section{REFERENCES}

[1] Hocoma, "Lokomat," February 8, 2018. [Online]. Available: https://www.hocoma.com/solutions/lokomat/

[2] J. Meuleman, E. Van Asseldonk, G. Van Oort, H. Rietman, and H. Van Der Kooij, "LOPES II - Design and Evaluation of an Admittance Controlled Gait Training Robot with Shadow-Leg Approach,' IEEE Transactions on Neural Systems and Rehabilitation Engineering, vol. 24, no. 3, pp. 352-363, 2016.

[3] Rewalk Robotics, "ReWalk Personal 6.0," February 8, 2018. [Online]. Available: http://rewalk.com/rewalk-personal-3/

[4] Ekso Bionics, "Ekso GT," March 7, 2018. [Online]. Available: https://eksobionics.com/eksohealth/products/

[5] Indego, "Indego Personal," March 7, 2018. [Online]. Available: http://www.indego.com/indego/en/Indego-Personal

[6] R. C. Browning, J. R. Modica, R. Kram, and A. Goswami, "The effects of adding mass to the legs on the energetics and biomechanics of walking," Medicine and Science in Sports and Exercise, vol. 39, no. 3 , pp. 515-525, 2007.

[7] A. Schiele, "Ergonomics of exoskeletons: Subjective performance metrics," 2009 IEEE/RSJ International Conference on Intelligent Robots and Systems, IROS 2009, pp. 480-485, 2009.

[8] A. B. Zoss, H. Kazerooni, and A. Chu, "Biomechanical Design of the Berkeley Lower Extremity Exoskeletong (BLEEX)," IEEE/ASME Transactions on Mechatronics, vol. 11, no. 2, pp. 128-138, 2006.

[9] S. B. Kesner, L. Jentoft, F. L. Hammond, R. D. Howe, and M. Popovic, "Design considerations for an active soft orthotic system for shoulder rehabilitation," Proceedings of the Annual International Conference of the IEEE Engineering in Medicine and Biology Society, EMBS, pp. 8130-8134, 2011

[10] Y. L. Park, B. R. Chen, D. Young, L. Stirling, R. J. Wood, E. Goldfield, and R. Nagpal, "Bio-inspired active soft orthotic device for ankle foot pathologies," IEEE International Conference on Intelligent Robots and Systems, pp. 4488-4495, 2011

[11] M. Wehner, B. Quinlivan, P. M. Aubin, E. Martinez-villalpando, M. Bauman, K. Holt, R. Wood, and C. Walsh, "A Lightweight Soft Exosuit for Gait Assistance," 2013 IEEE International Conference on Robotics and Automation (ICRA), pp. 3347-3354, 2013.

[12] A. T. Asbeck, R. J. Dyer, A. F. Larusson, and C. J. Walsh, "Biologically-inspired soft exosuit," IEEE International Conference on Rehabilitation Robotics, no. June, pp. 1-8, 2013.

[13] A. T. Asbeck, K. Schmidt, I. Galiana, D. Wagner, and C. J. Walsh, "Multi-joint soft exosuit for gait assistance," IEEE International Conference on Robotics and Automation, no. June, pp. 6197-6204, 2015

[14] L. N. Awad, J. Bae, K. O’Donnell, S. M. M. De Rossi, K. Hendron, L. H. Sloot, P. Kudzia, S. Allen, K. G. Holt, T. D. Ellis, and C. J. Walsh, "A soft robotic exosuit improves walking in patients after stroke," Science Translational Medicine, vol. 9, no. 400, p. eaai9084, 2017.

[15] B. T. Quinlivan, S. Lee, P. Malcolm, D. M. Rossi, M. Grimmer, C. Siviy, N. Karavas, D. Wagner, A. Asbeck, I. Galiana, and C. J. Walsh, "Assistance magnitude versus metabolic cost reductions for a tethered multiarticular soft exosuit," Science Robotics, vol. 2, no. 2, p. eaah4416, 2017.

[16] A. T. Asbeck, S. M. De Rossi, K. G. Holt, and C. J. Walsh, "A biologically inspired soft exosuit for walking assistance," The International Journal of Robotics Research, vol. 34, no. 6, pp. 744 762, 2015.

[17] J. Pratt, B. Krupp, C. Morse, and S. Collins, "The RoboKnee: an exoskeleton for enhancing strength and endurance during walking,' IEEE International Conference on Robotics and Automation, 2004. Proceedings. ICRA '04. 2004, no. April, pp. 2430-2435 Vol.3, 2004.

[18] J. C. Moreno, F. J. Brunetti, J. L. Pons, J. M. Baydal, and R. Barberà, "Rationale for multiple compensation of muscle weakness walking with a wearable robotic orthosis," Proceedings - IEEE International Conference on Robotics and Automation, vol. 2005, no. April, pp. 1914-1919, 2005.

[19] E. Richer and Y. Hurmuzlu, "A High Performance Pneumatic Force Actuator System: Part I-Nonlinear Mathematical Model," Journal of Dynamic Systems, Measurement, and Control, vol. 122, no. 3, p. 416, 2000

[20] _ _ "A High Performance Pneumatic Force Actuator System: Part II-Nonlinear Controller Design," Journal of Dynamic Systems, Measurement, and Control, vol. 122, no. 3, p. 426, 2000 